\title{
Hoher BMI verstärkt Thromboserisiko
}

Fragestellung: Haben übergewichtige Frauen ein höheres postoperatives Thromboserisiko als Normalgewichtige?

Hintergrund: Adipositas ist ein Risikofaktor für die Entstehung einer tiefen Beinvenenthrombose. Nun wurde erstmals in einer großen Studie untersucht, wie hoch das Thromboserisiko übergewichtiger Frauen ist.

Patienten und Methodik: Datenbasis für die englische Studie war die „Million Women Study“, ein Screeningprogramm des Nationalen Gesundheitsdienstes von England und Schottland, in dem 1170495 Frauen mit einem durchschnittlichen Alter von 56 Jahren 6 Jahre lang beobachtet wurden.

Ergebnisse: In dieser Zeit wurden 640288 Frauen wegen gastrointestinalen, gynäkologischen, orthopädischen und malignen Erkrankungen operiert, 4585
Parkin L, Sweetland S, Balkwill A et al. Body mass index, surge$r y$, and risk of venous thromboembolism in middle-aged women: a cohort study. Circulation 2012;125(15):1897-904. Frauen erlitten eine Venenthrombose. Auch ohne Operation stieg das Risiko für eine Beinvenenthrombose: Frauen mit einem BMI $>35 \mathrm{~kg} / \mathrm{m}^{2}$ hatten im Vergleich zu Frauen mit einem BMI zwischen 22,5 und

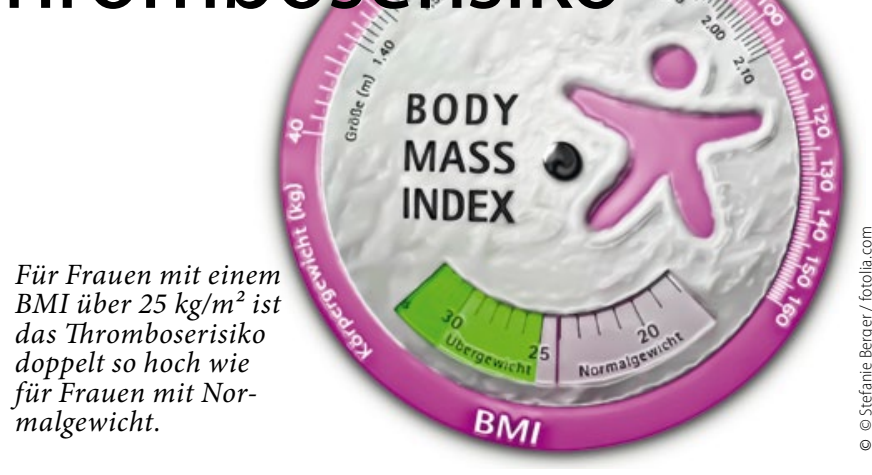

$25,0 \mathrm{~kg} / \mathrm{m}^{2}$ ein drei- bis vierfach erhöhtes Risiko. Im gleichen Ausmaß traf das auch für Lungenembolien zu. Frauen, die aufgrund einer Operation in ein Krankenhaus kamen, hatten im Vergleich zu Normalgewichtigen $48 \%$ bzw. 78\% häufiger Venenthrombosen, wenn sie übergewichtig bzw. adipös waren. In den 12 Wochen nach der Operation liefen Frauen mit einem BMI $>25 \mathrm{~kg} / \mathrm{m}^{2}$ doppelt so häufig Gefahr, an einer Venenthrombose zu erkranken, als diejenigen mit einem BMI $<25 \mathrm{~kg} / \mathrm{m}^{2}$.

Schlussfolgerungen: Übergewichtige Frauen sind stärker gefährdet an einer tiefen Beinvenenthrombose zu erkranken als Normalgewichtige. Eine Operation erhöht das Risiko zusätzlich.

\section{- Kommentar von Prof. Dr. med. Alfred Wirth}

\section{Das viele Fett drückt auf die Venen}

In den letzten Jahren konnten mehrere Untersuchungen überzeugend darlegen, dass nicht nur das arterielle, sondern auch das venöse Gefäßbett mit zunehmendem BMI einem erhöhten Krankheitsrisiko ausgesetzt ist. Große Kohortenstudien sowie eine Metaanalyse weisen aus, dass sowohl eine Beinvenenthrombose als auch eine Lungenembolie bei adipösen Menschen dreimal häufiger vorkommt. Die vorliegende Untersuchung zeigt nun, dass Übergewichtige und Adipöse zusätzlich deutlich häufiger postoperative venöse Komplikationen haben als Normalgewichtige. In anderen Studien wurde gezeigt, dass auch das postthrombotische Syndrom häufiger vorkommt und die Antikoagulation schwieriger ist.

Aus diesen Ergebnissen lassen sich folgende Schlussfolgerungen ziehen:

- Ein hoher BMI erhöht das Risiko für eine Venenthrombose.

- Ein hoher BMI bedingt häufiger Operationen, da die operativ zu behandelnden Krankheiten häufig mit Adipositas assoziiert sind (z.B. Cholezystolithiasis, degenerative Wirbelsäulen- und Gelenkerkrankungen, einige gynäkologische Krankheiten, manche Malignome).

- Wird operiert, tritt bei hohem BMI häufiger postoperativ eine Venenthrombose auf.

- Akute Venenthrombosen gehen bei Adipösen häufiger in ein postthrombotisches Syndrom über.

- Die Antikoagulation ist aus verschiedenen Gründen bei Adipösen häufiger unzureichend.

Die zugrundeliegenden Mechanismen für die Entstehung einer Venenthrombose bei vermehrter Körperfettmasse sind nicht ganz geklärt. Einfach zu verstehen ist, dass vermehrtes intraabdominales Fett die großen Bauchvenen komprimiert, damit den Druck in distalen Venen erhöht und deren Blutfluss reduziert. Adipöse haben im Mittel höhere Plasmakonzentrationen von Fibrinogen sowie einigen Gerinnungsfaktoren, auch die fibrinolytische Aktivität ist reduziert. Einige Produkte des Fettgewebes (Adipokine) sind inflammatorisch wirksam (z.B. TNF-alfa, Interleukine), was die Thrombosebereitschaft ebenfalls erhöht.

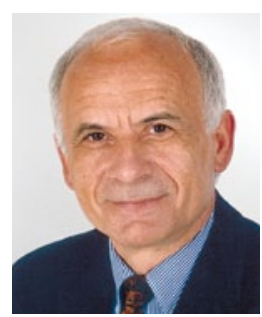

Prof. Dr. med. Alfred Wirth

Klinik Teutoburger Wald, Abt. Innere Medizin

Teutoburger-Wald-Str. 33

49214 Bad Rothenfelde 